\title{
A Function of Thermoplastic Starch Blends from Tapioca Waste Based on Mechanical Properties and Morphology
}

\author{
Yupawan Thongjun ${ }^{1, \mathrm{a}}$, Thiti Kaisone ${ }^{1, \mathrm{~b}}$, Pran Hanthanon ${ }^{1, \mathrm{c}}$, Chanon Wiphanurat ${ }^{1, \mathrm{~d}}$, Sumate \\ Ouipanich $^{1, e}$, Tarinee Nampitch ${ }^{1, f, *}$ \\ ${ }^{1}$ Department of Packaging and Materials Technology, Faculty of Agro-Industry, \\ Kasetsart University, Bangkok, Thailand
}

\begin{abstract}
The polymer was blended from tapioca residue and corn starch with 30 percent of glycerol by weight, which was prepared by a compression molding process at $135^{\circ} \mathrm{C}$ for 8 minutes. The formulation was monitored at 20,30, 40, 50 and 60 percent of the tapioca residue. The characterization provided mechanical properties, chemical composition and morphology. In the research, previous tapioca residue exhibited different functional groups in comparison with the current tapioca residue, which the previous tapioca residue consisted of high fiber content. A higher fiber content could improve the modulus and elongation to break dramatically, but it decreased the tensile strength. On the other hand, the fiber obtained the rough surface on the polymer matrix. In addition, the excess fiber could appear on the fiber aggregate.
\end{abstract}

Keywords: Thermoplastic starch, Tapioca residue, Compression molding, Fibers, Corn starch

\section{Introduction}

The world's waste pollutants from various sources are increasing, thus, causing a major environmental crisis. Petroleum-based polymer products, which are not biodegradable in the environment are one of the numerous problems. Therefore, many researchers around the world have become interested in biodegradable polymer and believe that products from this resource are going to prove to be environmentally-friendly. Many types of biodegradable polymer can be found at present; such as, PLA, PBAT, PBS, Chitin, etc. These kinds of biodegradable polymers replace some non-biodegradable polymers in various applications and areas; such as, mulch film, bioengineering, packaging, automotive parts including lifestyle tools like tableware, cups, electronic products, etc. However, the biodegradable polymers and bio-based polymers are of interest to researchers to search for knowledge to produce better work.

Tapioca or cassava is a common renewable material source in many countries like Thailand, Malaysia, Indonesia and Nigeria. The separation of soluble sugars and fibers of tapioca industrialization resulted in purified starch, residues, peel and bagasse [1]. It might also be observed that the residue is still rich in starch and fiber. Generally, tapioca residues after starch production comprises 45-60 percent of starch and about 15-20 percent of fiber [2]. Mostly, tapioca residues are applied to be an animal feed in the form of pellets or powder. From the benefit of the high starch and fiber content, research to expand the application of tapioca is required [3]. Nevertheless, the main limitation of tapioca residue is very poor in the formation process [4]. Blending tapioca residue with other polymers is an easy way to improve the properties. Tapioca residue reveals a high hydrophilic property through the high starch content. Based on good adhesion, polyester polymers, which contain a hydrophilic nature, are a potential candidate.

Starch appears in plants as granules with the main components of amylose and amylopectin polymer [5]. It is a completely biodegradable polymer, which can be obtained from various renewable resources. In addition, 
starch can be procured from byproducts via many processes; such as, harvesting and raw material industrialization [6]. Thermoplastic starch (TPS) is obtained by the destruction of the starch granule structure via a process with heat, shear forces in the presence of plasticizer [7]. Hence, TPS is a biodegradable, renewable and flexible material, which can be easily used in thermoplastic processing; such as, injection molding, extrusion of blow molding and compression molding [8]. TPS has a high glass transition temperature $\left(\mathrm{T}_{\mathrm{g}}\right)$ and is sensitive to humidity. These are the limitations of starch-based biodegradable materials [9]. The addition of glycerol as a plasticizer to cooperate with the starch improves the flexibility and processability. In addition, glycerol is widely known as one of the most suitable plasticizers of this material [10].

In this study, the properties of tapioca residue/TPS blends were investigated by different sources of tapioca residue. The investigation comprised mechanical, thermal and chemical properties.

\section{Materials and Methods}

\subsection{Materials}

Thermoplastic starch (TPS) was prepared from corn starch with SO2 more than $10 \mathrm{ppm}$. Amylose content $29.4 \mathrm{wt} \%$ and amylopectin content $22.5 \mathrm{wt} \%$ of Corn flour Super-Find. The plasticizer employed in this research work is $30 \%$ w/w of glycerol supplied by Quality Reagent Chemical. Fiber used for reinforcement is tapioca residues were supplied by Grace (Thailand) and RT AGRITE., LTD.

\subsection{Preparation of Thermoplastic Starch}

TABLE I: The Blends of Corn Starch, Plasticizer and Tapioca Residues by Grace (Thailand).

\begin{tabular}{cccc}
\hline \hline Sample name & Corn starch $(\%)$ & Tapioca residue $(\%)$ & Glycerol (\%) \\
\hline S80/G20 & 80 & 20 & 30 \\
S70/G30 & 70 & 30 & 30 \\
S60/G40 & 60 & 40 & 30 \\
S50/G50 & 50 & 50 & 30 \\
S40/G60 & 40 & 60 & 30 \\
\hline \hline
\end{tabular}

TABLE II: The blends of corn starch, plasticizer and tapioca residues by RT AGRITE., LTD.

\begin{tabular}{cccc}
\hline \hline Sample name & Corn starch $(\%)$ & Tapioca residue $(\%)$ & Glycerol $(\%)$ \\
\hline S80/R20 & 80 & 20 & 30 \\
S70/R30 & 70 & 30 & 30 \\
S60/R40 & 60 & 40 & 30 \\
S50/R50 & 50 & 50 & 30 \\
S40/R60 & 40 & 60 & 30 \\
\hline \hline
\end{tabular}

Impurities and contaminants were removed from the tapioca residues, followed by drying at $60^{\circ} \mathrm{C}$ for 12 hours in an oven to remove all moisture before grinding into a powder. A twin screw extruder was used to blend corn starch, tapioca residues and plasticizer at various ratios as shown in Table I and Table II. Temperature profiles were set from feed zone to die at 110,110 , and $120^{\circ} \mathrm{C}$ with screw speed maintained at $60 \mathrm{rpm}$. The extruder obtained from the die was cooled in the atmosphere and cut into pellets. The TPS blend was compressed at $135^{\circ} \mathrm{C}$ for 8 min using a compression molding machine.

\subsection{Mechanical Properties}

Tensile testing results determined tensile strength, elongation at break and modulus according to ASTM D638. Tensile testing was performed at $23^{\circ} \mathrm{C}$ using an Instron machine with crosshead speed of $1 \mathrm{~mm} / \mathrm{min}$ on at least five dumbbell-shaped specimens of each material type $\mathrm{V}$. 


\subsection{Fourier Transform Infrared (FT-IR) Spectroscopy}

Fourier transform infrared (FT-IR) spectroscopy was used in order to detect the presence of the functional group existed in tapioca residue. The range of wavenumber was attributed from 4000 to $400 \mathrm{~cm}-1$ in ATR mode.

\subsection{Scanning Electron Microscopy (SEM)}

The blends of thermoplastic starch and fiber were examined by using scanning electron microscope operated at $15 \mathrm{kV}$. The specimens were fractured under cryogenic condition in liquid nitrogen and the fractured surfaces vacuum were coated with paradium for scanning electron microscope.

\section{Results and Discussion}

\subsection{Mechanical Properties}

Tables III and IV show the data of the mechanical properties of the composite samples, which had different sources of tapioca residue. The different sources of the tapioca residue had an effect on the mechanical properties to the composite samples by the chemical composition of the tapioca residue. The tapioca residue by RT AGRITE., LTD had more modulus and less tensile strength than tapioca residue by Grace (Thailand) because of the high amount of fiber in the chemical composition. Thus, it can be assumed that the tapioca residue that had more fiber had a greater effect on the mechanical properties of the composites sample.

Table III: Mechanical Properties of the Blends Tapioca Residues by Grace (Thailand).

\begin{tabular}{cccc}
\hline \hline Sample name & Tensile strength $(\mathrm{MPa})$ & Elongation at Break $(\%)$ & Modulus $(\mathrm{MPa})$ \\
\hline S80/G20 & 7.46 & 2.50 & 219.64 \\
S70/G30 & 8.58 & 3.00 & 344.59 \\
S60/G40 & 4.50 & 1.00 & 357.33 \\
S50/G50 & 8.37 & 2.50 & 411.80 \\
S40/G60 & 10.98 & 3.50 & 438.60 \\
\hline \multicolumn{5}{r}{ Table IV: Mechanical Properties of the Blends Tapioca Residues by RT AGRITE., LTD. } \\
\hline \hline Sample name & Tensile strength (MPa) & Elongation at Break (\%) & Modulus (MPa) \\
S80/R20 & 5.53 & 2.54 & 598.66 \\
S70/R30 & 9.76 & 3.61 & 699.36 \\
S60/R40 & 4.79 & 2.67 & 646.91 \\
S50/R50 & 7.41 & 3.80 & 607.39 \\
S40/R60 & 10.50 & 3.86 & 573.51 \\
\hline \hline
\end{tabular}

\subsection{Fourier Transform Infrared (FT-IR) Spectroscopy}

The FTIR spectroscopy provided the functional groups as shown in Figure 1, which were detected by the absorption peak. Previously, the cassava starch residues presented a peak of $1333 \mathrm{~cm}-1$ and $1414 \mathrm{~cm}-1$ as showing the medium $\mathrm{OH}$ group blending of alcohol. The previous cassava residue peak could be observed similar to the current residues as $1076 \mathrm{~cm}-1$ and $1150 \mathrm{~cm}-1$ to present the $\mathrm{C}-\mathrm{O}$ stretching alcohol and C-O strong stretching aliphatic ester from the interaction between the cassava starch and fiber. Additionally, the same bands showing about $2920 \mathrm{~cm}-1,3285-3598 \mathrm{~cm}-1$ and $3629 \mathrm{~cm}-1$ were the $-\mathrm{CH}$ stretching alkane of the starch, $-\mathrm{OH}$ stretching intermolecular bonded, and -OH stretching free of water [11]. 


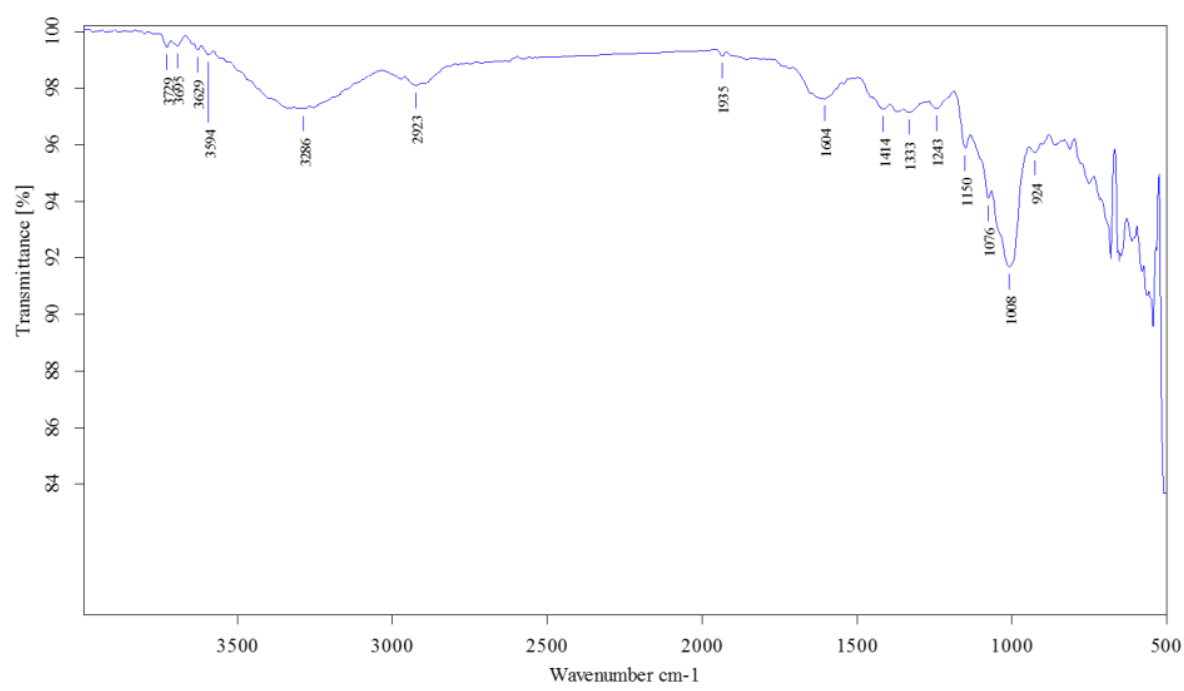

Fig. 1: FT-IR spectra of Tapioca residue

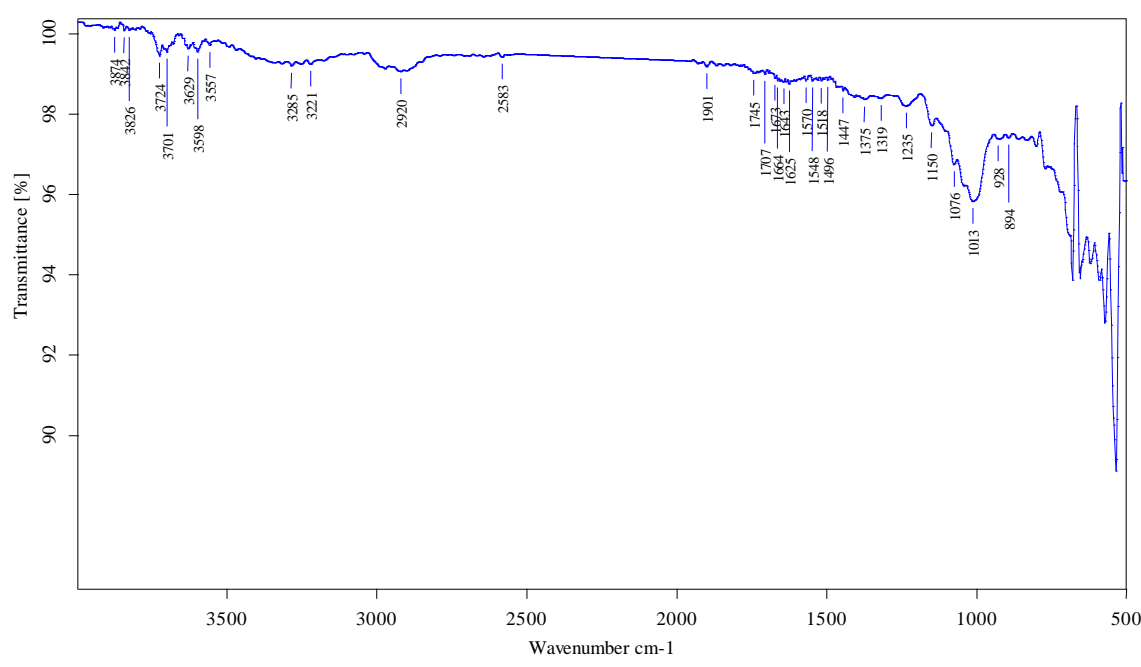

Fig. 2: FT-IR spectra of current tapioca residue

Moreover, the current cassava residue could be presented differently with the signal at $894 \mathrm{~cm}^{-1}$, the characteristic for the stretching vibration of the glycosidic bonds of the polysaccharides. In addition to this substance in terms of the fiber, this presented more peaks; such as, $1375 \mathrm{~cm}^{-1}, 1447 \mathrm{~cm}^{-1}, 1518 \mathrm{~cm}^{-1}, 1643 \mathrm{~cm}^{-1}$ and $1745 \mathrm{~cm}^{-1}$ that were $-\mathrm{CH}_{2}$, the $-\mathrm{CH}$ bending alkane group, $-\mathrm{CH}$ aliphatic, $-\mathrm{OH}$ the cellulose and carbonyl group of hemicellulose, respectively.

\subsection{Scanning Electron Microscopy (SEM)}

The fractured surface of the thermoplastic starch/fiber blends was done by a SEM. The SEM images shown in Figure 3 displayed the characterization of the blends with various tapioca residue contents of 20, 30, 40, 50 and $60 \backslash$ percent, respectively. The sample of the tapioca residue content of 80 percent could display a smooth surface and presented incomplete fiber dispersion. Additional tapioca residue could see the fiber aggregate decrease the mechanical properties, observing the S70/R30 of the tensile strength and elongation break from 9.76 MPa and 3.61 percent compared with S60/R40 of 4.79 MPa and 2.67 percent, respectively. However, the addition of the tapioca residue that was higher than 40 percent by weight could show the increment of the tensile strength and elongation at the break. The tapioca residue at 50 percent by weight could obviously show the fibers, which were permanent in the matrix. Moreover, the fibers exhibited a long fiber on the matrix surface. 

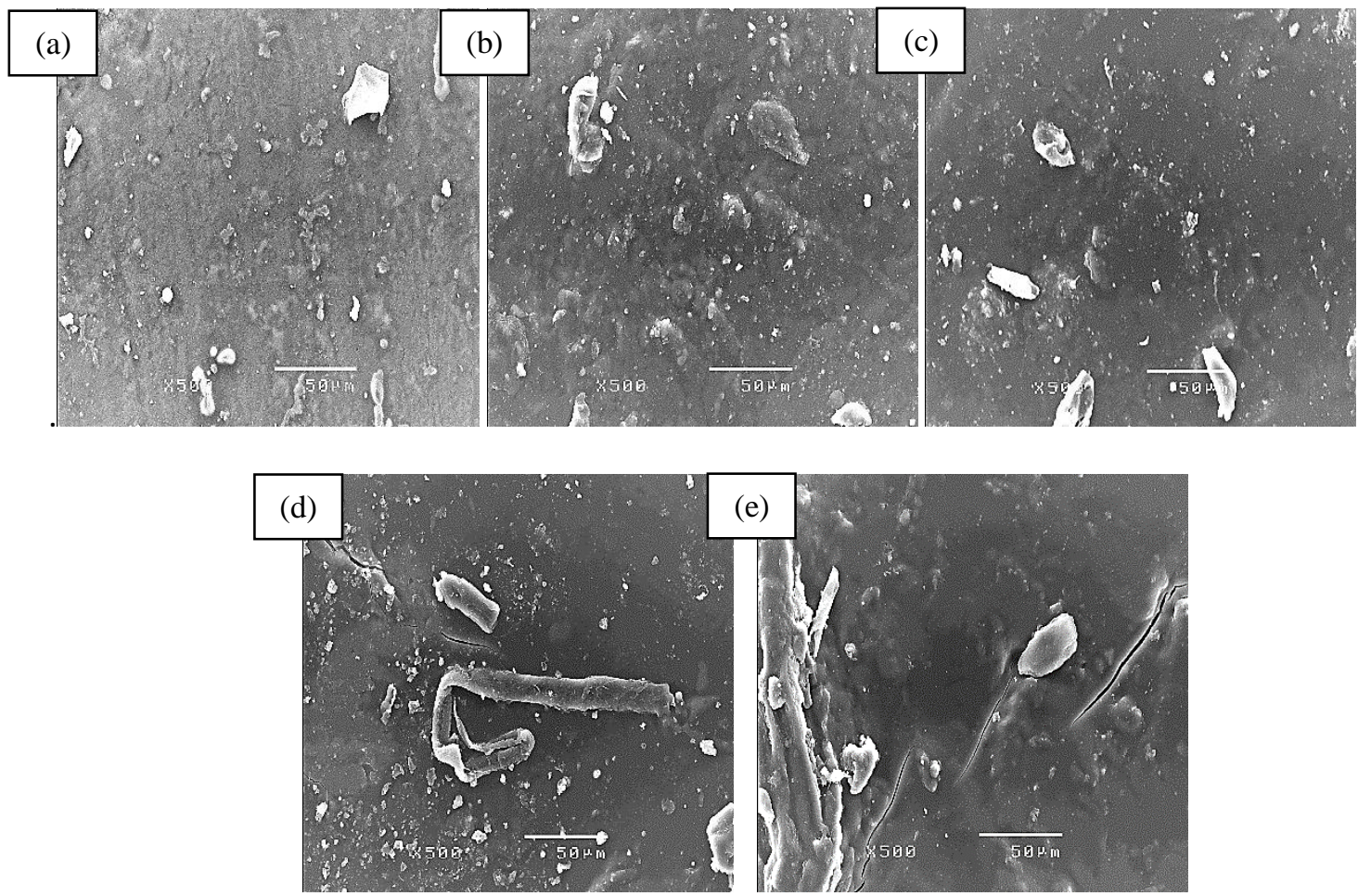

Fig. 3: SEM images of thermoplastic starch/tapioca residue blends with various tapioca contents (a) $20 \%$, (b) $30 \%$, (c) $40 \%$, (d) $50 \%$ and (e) $60 \%$

\section{Conclusions}

The blend samples were prepared by a twin screw extrusion and the specimens were produced by a compression molding process, which characterized the mechanical properties, chemical composition and morphological properties. Tensile properties decreased the increment of the tapioca residue by obtaining a rough surface. The functional group of the current tapioca residue presented the fiber composition to be more than the previous tapioca residue.

\section{Acknowledgements}

This research was financially supported by The Thailand Research Fund (TRF)

\section{References}

[1] L. R. F. SOUTO, M. CALIARI, M. S. S. JÚNIOR, F. A. FIORDA and M. C. GARCIA, "Utilization of residue from cassava starch processing for production of fermentable sugar by enzymatic hydrolysis," FOOD SCI TECH, vol. 37, pp. 19-24, June 2016.

[2] E. X. Leaes, E. Zimmermann, M. Souza, A. P. Ramon, E. T. Mezadri, V. D. Dal Prá, L. M. Terra, and M. A. Mazutti, "Ultrasound-assisted enzymatic hydrolysis of cassava waste to obtain fermentable sugars." BIOPROC BIOSYST $E N G$, vol. 115, pp. 1-6, May 2013.

https://doi.org/10.1016/j.biosystemseng.2013.02.001

[3] D. Sun and B. Yoo, "Effect of tapioca starch addition on rheological, thermal, and gelling properties of rice starch," LWT - FOOD SCI TECH, vol. 64, pp. 205-211, November 2015.

[4] M. Akrami, I. Ghasemi, H. Azizi, M. Karrabi and M. Seyedabadi, "A new approach in compatibilization of the poly(lactic acid)/thermoplastic starch (PLA/TPS) blends," CARBOHYD POLYM, vol. 144, pp. 254-262, February 2016. 
https://doi.org/10.1016/j.carbpol.2016.02.035

[5] H. C. Franklin, M. M. Yeison, L. M. Henry and P. Jorgelina, "Starch extraction potential from plantain peel waste," Journal of Environmental Chemical Engineering, vol. 5, pp. 4980-4985, October 2017.

https://doi.org/10.1016/j.jece.2017.09.034

[6] M. M. Marvizadeh, N. Oladzadabbasabadi, A. M. Nafchi and M. Jokar, "Preparation and characterization of bionanocomposite film based on tapioca starch/bovine gelatin/nanorod zinc oxide," LOGICAL MACROMOLECULES, vol. 99, pp. 1-7, June 2017.

https://doi.org/10.1016/j.ijbiomac.2017.02.067

[7] C. Bastioli, "Global status of the production of biobased packaging materials," Starch - Stärke, vol. 53, pp. 351-355, August 2001.

https://doi.org/10.1002/1521-379X(200108)53:8\%3C351::AID-STAR351\%3E3.0.CO;2-R

[8] A. M. Nafchi, M. Moradpour, M. Saeidi and A. K. Alias, "Thermoplastic starches: Properties, challenges, and prospects," Starch/Stärke, vol. 65, pp. 61-72, January 2013.

https://doi.org/10.1002/star.201200201

[9] P. Myllärinen, R. , Partanen, J. Seppälä and P. Forssell, "Effect of glycerol on behaviour of amylose and amylopectin films," CARBOHYD POLYM, vol. 50, pp. 355-361, December 2002.

https://doi.org/10.1016/S0144-8617(02)00042-5

[10] M. F. Cervera, M. Karjalainen, S. Airaksinen, J. Rantanen, K. Krogars, J. Heinamaki, A. I. Colarte and J. Yliruusi, "Physical stability and moisture sorption of aqueous chitosan-amylose starch films plasticized with polyols," EUR $J$ PHARM BIOPHARM, vol. 58, pp. 69-76, July 2004.

https://doi.org/10.1016/j.ejpb.2004.03.015

[11] K. O. Reddy, M. Shukla, C. U. Maheswari, and A. V. Rajulu, "Mechanical and physical characterization of sodium hydroxide treated Borassus fruit fibers," JFR, vol. 23, pp. 667-674. November 2014. 